\title{
REMOCIÓN DE NUTRIENTES EN AGUAS RESIDUALES DE UN MATADERO DE RESES USANDO UN REACTOR BIOLÓGICO SECUENCIAL
}

\section{NUTRIENTS REMOVAL FROM SLAUGHTERHOUSE WASTEWATER USING A SEQUENCING BATCH REACTOR}

\author{
Sedolfo Jose Carrasquero Ferrer , Daniela Carolina Marquina Gelvez², John Gerardo Soto López³, \\ Stefanía Viloria Rincón ${ }^{4}$, María Carolina Pire Sierra ${ }^{5}$, Altamira Rosa Díaz Montiel ${ }^{6}$
}

Fecha de recepción: 9 de abril de 2015

Fecha de aprobación: 21 de julio de 2015

Referencia: S.J. Carrasquero Ferrer, D.C. Marquina Gelvez, J.G. Soto López, S.V. Rincón, M.C. Pire Sierra, A.R. Díaz Montiel. (2015). Remoción de nutrientes en aguas residuales de un matadero de reses usando un reactor biológico secuencial. Ciencia e Ingeniería Neogranadina, 25 (2), pp. 43 - 60, D0l: http://dx.doi.org/10.18359/rcin.1431

\section{RESUMEN}

Se evaluó la remoción de nutrientes en un efluente cárnico utilizando un reactor por carga secuencial (SBR) a escala laboratorio. La investigación se llevó a cabo empleando aguas residuales de un matadero de reses ubicado en el Estado Zulia, Venezuela. El experimento se realizó en un reactor por carga secuencial con un volumen útil de $2 \mathrm{~L}$. El reactor fue operado bajo una secuencia operacional anaeróbica/aeróbica/anóxica, un tiempo de retención celular de 25 días y dos tiempos de retención hidráulica (11 y 15 h), generando dos tratamientos T1 y T2, respectivamente. Los parámetros medidos al inicio, durante y al final de cada ciclo evaluado fueron demanda química de oxígeno total (DQOT), nitrógeno total Kjeldahl (NTK), nitrógeno amoniacal ( $\mathrm{N}-\mathrm{NH}_{4^{+}}$), nitritos ( $\left.\mathrm{N}-\mathrm{NO}_{2}\right)^{-}$, nitratos ( $\left.\mathrm{N}-\mathrm{NO}_{3}\right)$, $\mathrm{pH}$, alcalinidad total y fósforo total (PT). Los resultados de las remociones de las variables se compararon mediante un análisis de varianza

\footnotetext{
1. Ing. Químico. Msc en Ingeniería Ambiental. Profesor Agregado. Departamento de Ingeniería Sanitaria y Ambiental. Universidad del Zulia. Maracaibo, Venezuela. scarrasquero@fing.luz.edu.ve

2. Ingeniera Civil. Departamento de Ingeniería Sanitaria y Ambiental. Universidad del Zulia. Maracaibo, Venezuela, dani.carol152@gmail.com

3. Ingeniero Civil. Departamento de Ingeniería Sanitaria y Ambiental. Universidad del Zulia. Maracaibo, Venezuela, jnsolo@hotmail.com

4. Ingeniera Civil. Departamento de Ingeniería Sanitaria y Ambiental. Universidad del Zulia. Maracaibo, Venezuela, stefaniav04@gmail.com

5. Ingeniero Químico. Dra. en Ingeniería Ambiental. Profesora Asociada. Programa de Ingeniería Agroindustrial. Universidad Centroccidental Lisandro Alvarado. Barquisimeto, Venezuela. carol_pire@yahoo.es

6. Ingeniero Civil. Dra. en Ingeniería y Procesos del Medio Ambiente. Profesora Titular. Departamento de Ingeniería Sanitaria y Ambiental. Universidad del Zulia. Maracaibo, Venezuela, adiaz@fing.luz.edu.ve
} 
y separación de medias a través de la prueba de Tukey, utilizando el programa estadístico Statistix 9.0. Las aguas residuales provenientes de la matanza de reses presentaron gran variabilidad en sus características fisicoquímicas, viéndose reflejado en los altos valores de desviación estándar que se obtuvieron. Los dos tratamientos aplicados al efluente industrial del matadero de reses en el reactor por carga secuencial permitieron la remoción simultánea de nutrientes y materia orgánica, debido a la flexibilidad del reactor para adaptarse a distintas condiciones de operación, obteniendo remociones superiores al $95 \%$ para la DQO, $69 \%$ para el NT y $29 \%$ para el PT.

Palabras clave: Efluente Cárnico, Reactor por Carga Secuencial (SBR), Remoción de Nutrientes.

\section{ABSTRACT}

Simultaneous removal of organic matter, nitrogen and phosphorus in slaughterhouse wastewater was evaluated using a sequential batch reactor (SBR) at laboratory scale. The study was carried out using wastewater from an abattoir located in the Zulia state, Venezuela. The experiment was conducted using a completely randomized design, a cell retention time of 25 days, an anaerobic-aerobic-anoxic sequence, and two hydraulic retention times of 11 and 15 hours. Parameters were measured at the beginning, during and at the end of each cycle, and those were chemical oxygen demand (COD), biochemical oxygen demand (BOD), total Kjeldahl nitrogen (TKN), ammonia nitrogen ( $\left.\mathrm{N}-\mathrm{NH}_{4^{+}}\right)$, nitrite $\left(\mathrm{N}-\mathrm{NO}_{2}-\right)$, nitrates $\left(\mathrm{N}-\mathrm{NO}_{3}-\right)$, $\mathrm{pH}$, alkalinity, total phosphorus (TP). The results of the removal of variables were compared using analysis of variance and mean separation by Tukey's test, using the statistical program Statistix 9.0. The two treatments applied to slaughterhouse wastewater in reactor allowed the simultaneous removal of nutrients and organic matter, due to the flexibility of the reactor to suit different operating conditions, obtaining removal of $95 \%$ for COD , $69 \%$ for TN and $29 \%$ for TP.

Keywords: Nutrient Removal, Sequencing Batch Reactor (SBR), Slaughterhouse Wastewater.

\section{INTRODUCCIÓN}

Los efluentes industriales se caracterizan por ser de composición muy diversa y compleja, debido a que se generan de sistemas productivos diferentes, donde las materias primase insumosqueseutilizan le proporcionan características particulares a cada tipo de agua residual generada [1]. Además, el volumen y la concentración de los contaminantes en los efluentes industriales son considerablemente mayores a los efluentes domésticos, por lo que resulta conveniente la aplicación de un sistema de tratamiento que pueda adaptarse al gran número de contaminantes que se encuentran contenidos en estos efluentes [2].

Las descargas provenientes de la agroindustria, específicamente del proceso de matanza de reses, presentan un gran potencial contaminante que varía dependiendo del número de animales sacrificados diariamente, 
la dieta del animal faenado, la recolección de productos y subproductos, la racionalidad en el uso del recurso hídrico dentro del proceso, y muchas veces la descarga o no del agua residual proveniente de las instalaciones sanitarias de la planta [3].

Los efluentes provenientes del proceso de matanza contienen elevadas concentraciones de materia orgánica, coliformes totales, sólidos suspendidos, grasas, nitrógeno y fósforo, representando una mezcla compleja de proteínas, compuestos orgánicos y grasas. La problemática asociada a la presencia de los nutrientes ( $\mathrm{N}$ y $\mathrm{P}$ ) radica ante todo en la eutrofización de corrientes naturales, entre las que se cuentan los lagos y embalses, lo que genera un fuerte impacto ambiental que pone en riesgo la vida en los ecosistemas acuáticos [4-6].

Es por esto que en la actualidad se ha visto la necesidad de remover simultáneamente la materia orgánica, el nitrógeno y el fósforo en los efluentes provenientes de los mataderos de reses, realizándose exitosamente mediante la aplicación de tratamientos biológicos con sistemas no convencionales de lodos activados, como los reactores por carga secuencial (SBR) [7-10].

Estos reactores, además de ser efectivos en la eliminación simultánea de nutrientes, son más económicos y generan menor cantidad de subproductos que los sistemas biológicos convencionales [11].

El objetivo de esta investigación fue evaluar la tratabilidad del agua residual de un matadero de reses ubicado en el Estado Zulia, mediante el uso de un reactor por carga secuencial a escala laboratorio, aplicando dos estrategias operacionales para lograr la remoción simultánea de materia orgánica, nitrógeno y fósforo en este tipo de efluentes, analizando la descarga del sistema de tratamiento con respecto a lo establecido en las normas de descargas venezolanas contempladas en el Decreto 883 [12].

\section{MATERIALES Y MÉTODOS}

Los estudios se llevaron a cabo utilizando aguas residuales de un matadero de reses (Bos taurus) ubicado en el Estado Zulia, Venezuela. La captación del agua residual se hizo de manera manual a través de un muestreo aleatorio simple, tomando las aguas residuales en una tanquilla ubicada en las afueras del matadero, en envases de polietileno de alta densidad de $25 \mathrm{~L}$, limpios y de color oscuro.

Para el tratamiento del efluente del matadero de reses se utilizó un reactor cilíndrico de $14,5 \mathrm{~cm}$ de diámetro y $26 \mathrm{~cm}$ de alto, con un volumen de $4 \mathrm{~L}$. El volumen de operación fue de $2 \mathrm{~L}$, del cual un $30 \%$ correspondió a lodo activado y el resto del volumen correspondió al efluente industrial.

El reactor funcionó de manera automatizada para la carga y descarga del agua a tratar mediante un sistema de bombeo con bombas peristálticas (Cole-Parmer, modelos 7720260 y 77201-60) y dispositivos reguladores de tiempo (Excelline, modelo GTC-E-120AS). El reactor por carga secuencial también dispuso de sistemas automatizados de agitación mecánica y suministro de oxígeno (figura 1).

Dicho sistema de agitación mecánica estuvo compuesto de un motor monofásico (General Electric, modelo WR60X165) de $15 \mathrm{~W}$ de potencia y $1.300 \mathrm{rpm}$, ensamblado a un eje que dispone de un aspa de dos alas que fue 


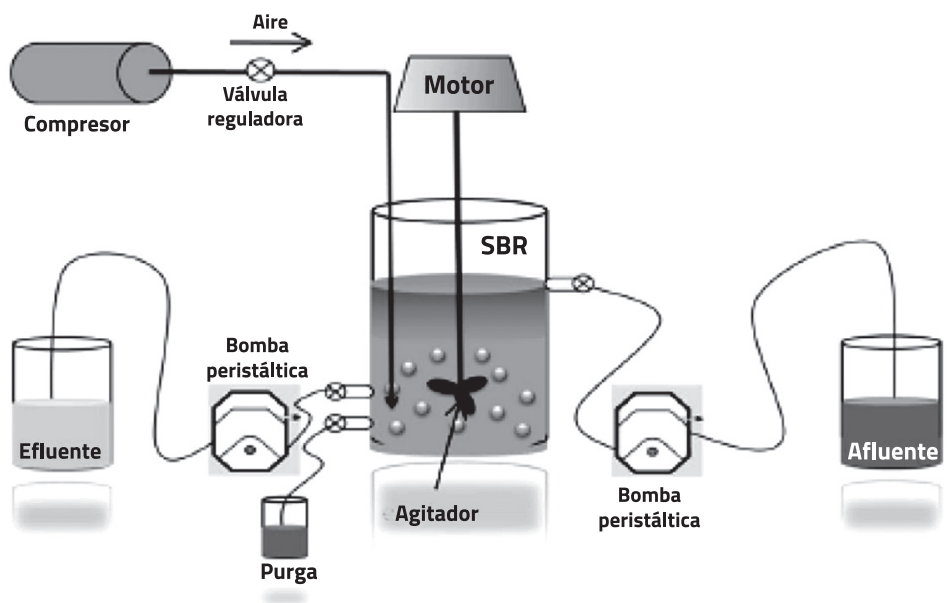

Figura 1. Descripción ilustrada del reactor por carga secuencial (SBR).

sumergido dentro del agua residual y del lodo (licor mezcla). Las revoluciones de los motores se mantuvieron en 300 rpm por medio de un potenciómetro [13].

El oxígeno se suministró a través de un compresor de aire (SeaStar, modelo HX-30820), el cual estuvo conectado a una tubería flexible transparente de $5 \mathrm{~mm}$ enlazada en serie con un dispositivo difusor dispuesto en el fondo del reactor de $45 \mathrm{~cm}$ de largo y 4 $\mathrm{mm}$ de diámetro. Este dispositivo se encargó de suministrar aire en forma ascendente a la mezcla contenida en el reactor.

La biomasa fue obtenida de un sistema biológico de tratamiento de efluentes cárnicos. Una vez inoculado el reactor con la biomasa, se sometió a un proceso de estabilización a las primeras condiciones operacionales que se experimentaron: tiempo de retención celular de 25 días, tiempo de retención hidráulica de 11 horas y una secuencia operacional anaeróbicaaeróbica-anóxica. El final de la estabilización para el comienzo del muestreo se determinó cuando se alcanzaron valores estables de remoción de DQO a la salida del reactor, superiores a $50 \%$ y buenas características de sedimentabilidad en el lodo.

El efluente cárnico antes de ser alimentado al reactor fue sometido a un pre-tratamiento, el cual consistió en ajustar el nivel de $\mathrm{pH}$ del agua a niveles óptimos (6,5 a 8,5 unidades). El ajuste de $\mathrm{pH}$ se hizo con hidróxido de sodio $(\mathrm{NaOH}) 6,0$ $\mathrm{N}$. Los pH obtenidos favorecieron el crecimiento de los microorganismos específicos que intervienen en los procesos de degradación y estabilización de la materia orgánica en los tratamientos [14].

Deigual manera, paramejorar las características de las aguas residuales y facilitar su tratamiento biológico, los sólidos suspendidos en el afluente fueron removidos por separación con una malla No. 20, para evitar que pudieran causar la obturación de tuberías y bombas del sistema automatizado de carga y descarga de los reactores.

El proceso de tratamiento del efluente constó de cuatro etapas: alimentación, reacción, sedimentación y descarga. En la fase anaeróbica (An) no se proporcionó ni aire ni agitación, en 
la aeróbica (Ae) se inyectó aire y agitación al sistema, para obtener un mezclado entre el lodo y el agua residual; en la fase anóxica (Ax) se proporcionó solo agitación al agua residual suministrada al reactor; mientras que en la fase de sedimentación, el lodo decantó por acción de la gravedad y se separó del agua residual tratada, donde se obtuvo un sobrenadante clarificado; en la etapa de vaciado se retiró el agua residual tratada (sobrenadante) a través del sistema de descarga y en la etapa de purga se realizó la extracción del lodo en exceso.

La entrada del afluente y la descarga del efluente del reactor se hizo a través de una tubería flexible (Masterflex 06409-147) de $6 \mathrm{~mm}$ de diámetro, conectada al reactor empleando bombas peristálticas de un solo sentido de flujo, las cuales se mantuvieron a caudal constante de $93 \mathrm{~mL} / \mathrm{min}$ para el llenado y descarga. La extracción del licor mezcla se efectúo a través de una tubería flexible (Masterflex 06409-147) de 6 mm de diámetro conectada al reactor.

Durante el estudio se trabajó con un tiempo de retención celular de 25 días, mientras que el tiempo de retención hidráulica varió, primero se utilizó 11 horas y después un tiempo de 15 horas. Esto dio origen a dos tratamientos, T1 y T2. Para el tratamiento T1, la duración de las fases fue: $1 \mathrm{~h}$ para la fase anaeróbica, $7 \mathrm{~h}$ en la fase aeróbica y $2 \mathrm{~h}$ de fase anóxica, mientras que en el tratamiento T2 la fase anaeróbica fue de 1,5 h, la fase aeróbica de 10,0 h y la fase anóxica de 2,5 h. Para todos los tratamientos el tiempo de las fases de llenado, sedimentación y descarga es constante $(0,25 h ; 0,50$ h y 0,25 $h$, respectivamente).

Los parámetros medidos a la entrada, salida de cada una de las fases de la etapa de reacción y al finalizar el tratamiento de cada ciclo fueron los siguientes: $\mathrm{pH}$ (4500-B), $\mathrm{DBO}_{5-20}$ (SM 5210), $\mathrm{DQO}_{\mathrm{T}}$ y $\mathrm{DQO}_{S}$ (5220-C), NTK (4500-Norg-B), $\mathrm{N}-\mathrm{NH}_{4^{+}}\left(4500-\mathrm{NH}_{3} \mathrm{~B}\right), \mathrm{N}-\mathrm{NO}_{2^{-}}\left(4500-\mathrm{NOZ}_{2^{-}}\right.$ $-\mathrm{B}), \mathrm{N}-\mathrm{NO}_{3^{-}}\left(4500-\mathrm{NO}_{3}-\mathrm{D}\right), \mathrm{NT}$ (4500-Norg-B), PT (4500-P-C), P-PO ${ }_{4-3}^{-3}(4500-P-C)$, alcalinidad total (2320 B), SS (2540-F), SSV (2540-E) y SST (2540-D) según los procedimientos establecidos en el Método Estándar [15]. Para la determinación de la $\mathrm{DQO}_{s}$, las muestras fueron previamente filtradas por una membrana de celulosa y un tamaño de poro de 0,45 $\mu \mathrm{m}$ (Whatman).

El experimento se condujo mediante un diseño completamente al azar, con dos tratamientos de doce repeticiones cada uno; los resultados de las remociones de las variables se compararon mediante un análisis de varianza y separación de medias a través de la prueba de Tukey, utilizando el programa estadístico Statistix 9.0. Los resultados del resto de las variables evaluadas fueron presentados mediante estadística descriptiva señalando los valores de tendencia central (media) y su dispersión (desviación estándar).

\section{RESULTADOS Y DISCUSIÓN}

\subsection{CARACTERIZACIÓN DEL EFLUENTE DEL MATADERO DE RESES}

El agua residual industrial del matadero está formada por una mezcla de agua-sangre, desechos ruminales producto del lavado de las vísceras, grasas y estiércol; la misma es descargada junto con las aguas residuales provenientes de las instalaciones sanitarias de la planta de matanza. En la tabla 1 se presentan los resultados obtenidos durante 
la caracterización del efluente industrial, reportando los valores medios y su dispersión expresada a través de la desviación estándar; de igual manera, se indican los límites establecidos por la norma venezolana de descarga a los cuerpos de agua [12].

El efluente industrial proveniente del matadero de reses se caracterizó por presentar un contenido de materia orgánica total, medida como $\mathrm{DQO}_{\text {T, }}$ que osciló entre 7.536 y 25.676 $\mathrm{mg} / \mathrm{L}$, con una media de $16.606 \mathrm{mg} / \mathrm{L}$, excediendo lo establecido por la norma venezolana de descarga en los cuerpos de agua, que contempla un valor máximo de 350 $\mathrm{mg} / \mathrm{L}[11]$. Debido a que el matadero en estudio no realiza ningún proceso de separación y/o recuperación de la sangre, los valores de $\mathrm{DQO}_{\mathrm{T}}$ obtenidos en el presente trabajo son superiores a los reportados por Kundu et al. [16], Ramón y Maldonado [17] y Bazrafshan et al. [18], quienes reportaron valores de 6.512; 5.364 y $5.817 \mathrm{mg} / \mathrm{L}$, respectivamente.

La recuperación de la sangre se da en los mataderos que poseen el equipo correspondiente para su separación y procesamiento con la finalidad de elaborar morcillas y alimentos para animales, pero en los mataderos que no realizan esta operación, la sangre es descargada directamente al colector. La recuperación de la sangre significa una disminución del 42 \% de la carga orgánica total de este tipo de vertidos. El potencial

Tabla 1. Características fisicoquímicas del efluente industrial.

\begin{tabular}{|c|c|c|}
\hline Parámetro & $\begin{array}{c}\text { Valor } \\
\text { (media } \pm \mathrm{DE})\end{array}$ & $\begin{array}{l}\text { Límites establecidos por la norma de descarga } \\
\qquad \text { a los cuerpos de agua }\end{array}$ \\
\hline $\mathrm{pH}$ & $6,36 \pm 0,07$ & $6-9$ \\
\hline Alcalinidad total & $356 \pm 159$ & - \\
\hline $\mathrm{DQO}_{\mathrm{T}}$ & $16.606 \pm 9.070$ & 350 \\
\hline $\mathrm{DBO}_{5,20}$ & $8.113 \pm 1.684$ & 60 \\
\hline NTK & $646,84 \pm 259,33$ & - \\
\hline $\mathrm{N}-\mathrm{NH}_{4}{ }^{+}$ & $319,58 \pm 128,12$ & - \\
\hline $\mathrm{N}-\mathrm{NO}_{2}^{-}$ & ND & $\mathrm{N}-\mathrm{NO}_{2}{ }^{-}+\mathrm{N}-\mathrm{NO}_{3}{ }^{-}=10$ \\
\hline $\mathrm{N}-\mathrm{NO}_{3}^{-}$ & ND & $\mathrm{N}-\mathrm{NO}_{2}{ }^{-}+\mathrm{N}-\mathrm{NO}_{3}^{-}=10$ \\
\hline NT & $646,84 \pm 259,33$ & 40 \\
\hline SST & $600 \pm 170$ & 80 \\
\hline SSV & $450 \pm 156$ & - \\
\hline $\mathrm{PT}$ & $10,9 \pm 0,9$ & 10 \\
\hline $\mathrm{DBO}_{5,20} / \mathrm{DQO}_{\mathrm{T}}$ & 0,49 & - \\
\hline $\mathrm{DQO}_{\mathrm{T}} / \mathrm{N}-\mathrm{NH}_{4}{ }^{+} / \mathrm{P}_{\mathrm{T}}$ & $100: 1,92: 0,07$ & - \\
\hline
\end{tabular}


contaminante de las aguas residuales de un matadero de reses varía en función del número y tipo de animales sacrificados, el volumen de agua utilizado y el producto recuperado [19].

Otros autores como Nakhla et al. [20] y Sunder y Satyanarayan [19] obtuvieron concentraciones de $\mathrm{DQO}_{\mathrm{T}}$ de 16.800 y $24.750 \mathrm{mg} / \mathrm{L}$, respectivamente, valores que se encuentran dentro del rango de concentración de $\mathrm{DQO}_{\mathrm{T}}$ obtenido en el presente estudio.

En relación con la cantidad de materia orgánica contenida en el efluente del matadero de reses, medida como $\mathrm{DBO}_{5,20^{\prime}}$ el valor obtenido durante la caracterización osciló entre 6.429 y $9.796 \mathrm{mg} / \mathrm{L}$; con una media de $8.113 \mathrm{mg} / \mathrm{L}$, encontrándose dicho parámetro por encima del límite establecido por la norma venezolana para descarga en los cuerpos de agua [12].

La biodegradabilidad de los efluentes industriales está determinada por la relación existente entre los parámetros $\mathrm{DBO}_{5,20}$ y $\mathrm{DQO}_{\mathrm{T}}$. Para el efluente del matadero de reses en estudio, este valor resultó ser de 0,49 ; lo que indica que el efluente es moderadamente biodegradable [21]. El índice de biodegradabilidad obtenido en esta investigación se encontró cercano a los reportados por Kundu etal. [16].y Bazrafshan et al. [18]. Estos autores trabajaron con efluentes de matadero de reses y obtuvieron valores de 0,50 y 0,43 , respectivamente.

Con respecto al $\mathrm{pH}$, los valores obtenidos en el agua residual estuvieron comprendidos entre 6,29 y 6,43 unidades, caracterizándose el efluente por presentar una alcalinidad total que osciló entre 197 y 516 mg/L, con un valor medio de $357 \mathrm{mg} / \mathrm{L}$, resultados muy cercanos a los reportados por Díaz et al. [14], quienes trabajaron con efluentes industriales camaroneros y obtuvieron un valor medio de alcalinidad a la entrada de un reactor por carga secuencial de $304 \mathrm{mg} / \mathrm{L}$ de $\mathrm{CaCO}_{3}$.

En relación con el $\mathrm{NTK}, \mathrm{N}-\mathrm{NH}_{4^{+}}$y $\mathrm{PT}$, los resultados obtenidos fueron 646,$84 ; 319,58$ y $10,86 \mathrm{mg} / \mathrm{L}$, respectivamente, observando que el efluente del matadero de reses posee un alto contenido de nitrógeno orgánico, el cual representó el 50,59 \% del nitrógeno total.

La relación DQOT/N- $\mathrm{NH}_{4^{+}} / \mathrm{PT}$ en el efluente resultó ser de 100:1,92:0,07; la cual es menor a la reportada por Nacheva et al. [22], quienes obtuvieron una relación igual a 100:9,6:0,48; sugiriendo 100:1,2:0,17 como valores óptimos para la aplicación de procesos biológicos en el tratamiento de aguas residuales industriales de mataderos de reses.

En los resultados obtenidos se puede notar la gran variabilidad de las características fisicoquímicas del efluente, valores que se encontraron fuera de los límites establecidos por la norma venezolana de descarga en los cuerpos de agua [12], a excepción del pH que se mantuvo dentro del rango impuesto por la normativa. Esta variación pudo verse reflejada por los altos valores de desviación estándar alcanzados durante los muestreos.

\subsection{DESEMPEÑO DE LAS VARIABLES EVALUADAS EN EL SBR}

En la tabla 2 se presentan los valores de concentración promedio de materia orgánica, expresados en términos de $\mathrm{DQO}_{\mathrm{T}}$, a la entrada, al final de cada fase y a la salida del reactor, así como también los porcentajes de remoción que se obtuvieron para cada tratamiento. Se puede observar que las concentraciones promedio de materia orgánica a la entrada 
Tabla 2. Valores promedios de las concentraciones, velocidades de remoción de la carga orgánica y porcentajes de remoción de $\mathrm{DQO}_{\mathrm{T}}$ en cada tratamiento aplicado en el reactor SBR.

\begin{tabular}{|c|c|c|c|}
\hline Variable & Fase & T1 & $\mathrm{T} 2$ \\
\hline \multirow{5}{*}{$\mathrm{DQO}_{\mathrm{T}}(\mathrm{mg} / \mathrm{L})$} & Carga & $12.756 \pm 3.839$ & $13.522 \pm 4.910$ \\
\hline & Fin Anaeróbica & $8.383 \pm 306$ & $6.933 \pm 185$ \\
\hline & Fin Aeróbica & $938 \pm 157$ & $533 \pm 113$ \\
\hline & Fin Anóxica & $608 \pm 238$ & $485 \pm 205$ \\
\hline & Salida & $582 \pm 165$ & $517 \pm 151$ \\
\hline \multicolumn{2}{|r|}{ \% Remoción DQO } & $95,3^{\mathrm{a}} \pm 0,8$ & $95,4^{\mathrm{a}} \pm 1,7$ \\
\hline \multicolumn{2}{|c|}{$\begin{array}{l}\text { Velocidad de la remoción de la carga orgánica } \\
\qquad\left(\mathrm{mg} \mathrm{O}_{2}{ }^{\left.* L^{-1 *} \mathrm{~min}^{-1}\right)}\right.\end{array}$} & $20,2^{\mathrm{a}} \pm 6,00$ & $15,5^{b} \pm 5,60$ \\
\hline \multicolumn{2}{|c|}{$\begin{array}{l}\text { Velocidad específica de remoción de carga orgánica }\left(\mathrm{mg} \mathrm{O}_{2}{ }^{*}\right. \\
\left.\qquad \mathrm{mg} \text { Biomasa }{ }^{*} \mathrm{~min}^{-1}\right)\end{array}$} & $0,0104^{\mathrm{a}} \pm 0,0031$ & $0,0051^{b} \pm 0,0019$ \\
\hline
\end{tabular}

del primer y segundo tratamiento, T1 y T2, fueron de $12.756 \mathrm{mg} / \mathrm{L}$ y $13.522 \mathrm{mg} / \mathrm{L}$, respectivamente, mientras que a la salida del reactor se obtuvieron unos valores promedio de $583 \mathrm{mg} / \mathrm{L}$ para T1 y $517 \mathrm{mg} / \mathrm{L}$ para T2. Se encontró que los porcentajes de remoción obtenidos para ambos tratamientos fueron estadísticamente similares ( $p>0,05)$.

Las eficiencias de remoción de $\mathrm{DQO} \mathrm{T}_{\mathrm{T}}$ alcanzadas en el reactor por carga secuencial, tanto para el tratamiento T1 como para T2, se encontraron dentro del rango reportado para otros sistemas de tratamiento biológico. Autores como Massé y Masse [6] lograron una remoción entre el 90 y $96 \%$ de la $\mathrm{DQO}_{\text {T }}$ en un efluente de un matadero de cerdos, usando un reactor por carga anaeróbico con un TRH de 2 días, mientras que Ramón y Maldonado [17] quienes aplicaron un tratamiento biológico combinado que consistió en un filtro anaeróbico de flujo ascendente y un sistema biodiscos en efluentes de matadero de reses, lograron porcentajes de remoción comprendidos entre 88 y $95 \%$ para TRH entre 3,33 y 2,10 días.

Durante esta investigación se lograron altos porcentajes de remoción, superiores a 95\% mediante la aplicación de un tratamiento biológico, que al compararlos con los obtenidos por procesos fisicoquímicos demuestran la eficiencia del reactor en el tratamiento de este tipo de efluentes. Bazrafshan et al. [18] trataron efluentes de matadero de reses mediante procesos de coagulación y el uso de policloruro de aluminio, en conjunto con un proceso de electrocoagulación usando electrodos de aluminio, con lo que se logró obtener una eficiencia de remoción del $96 \%$.

La figura 2 corresponde a los perfiles de la $\mathrm{DQO}_{\mathrm{T}}$ durante la fase de reacción para los dos tratamientos realizados. Se puede observar que gran parte de la remoción de la materia orgánica ocurrió durante las fases anaeróbica y aeróbica, mientras que la $\mathrm{DQO}_{\mathrm{T}}$ durante la 

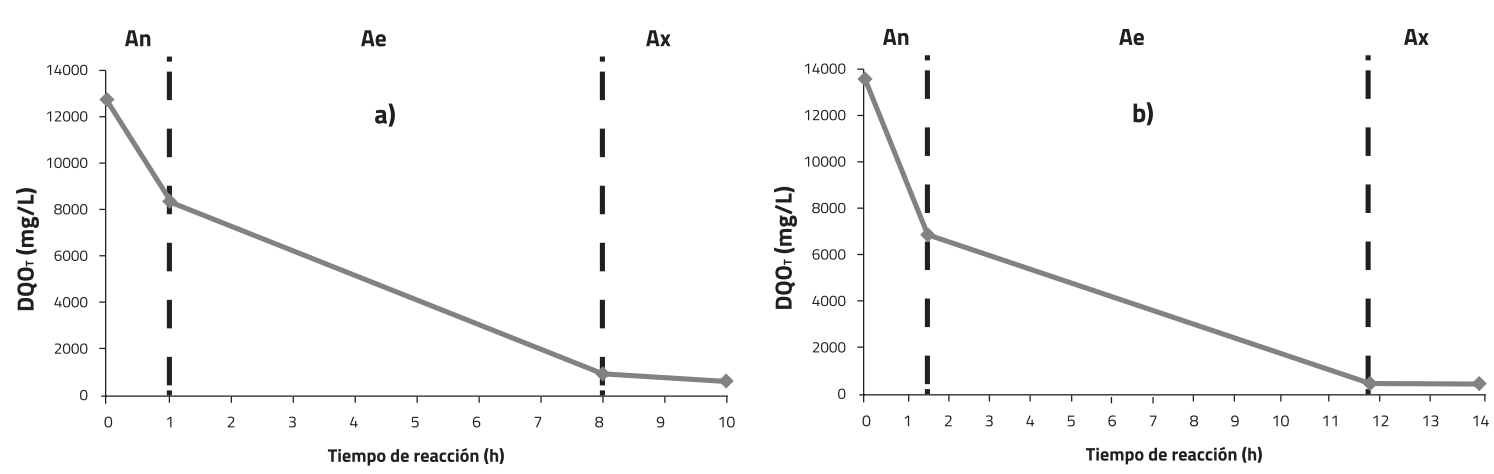

Figura 2. Evolución de la $\mathrm{DQO}_{\mathrm{T}}$ durante los tiempos de reacción de un ciclo de operación del reactor para los tratamientos T1 (a) y T2 (b)

fase anóxica permaneció casi constante. En la figura 2a, referente al primer tratamiento, se muestra una concentración promedio de $\mathrm{DQO}_{\mathrm{T}}$ de $12.756 \mathrm{mg} / \mathrm{L}$ a la entrada del reactor. Luego de 1 hora de fase anaeróbica, parte de la materia orgánica fue consumida por los microorganismos anaeróbicos logrando un $34,3 \%$ de remoción, y durante las siguientes 7 horas de fase aeróbica ocurrió la mayor remoción de materia orgánica de 88,8 \%. Sin embargo, para las últimas 2 horas de fase anóxica se tiene una remoción menor, de tan solo el $4,3 \%$.

En la figura 2b, referente al segundo tratamiento, se puede observar que a medida que se aumentó el tiempo de reacción durante las fases anaeróbica y aeróbica, el porcentaje de remoción de materia orgánica fue mayor durante dichas fases.

Aunque los porcentajes de remoción que se lograron en esta investigación superaron el $95 \%$, los valores medios de $\mathrm{DQO}_{\text {T }}$ que se obtuvieron a la salida del reactor SBR fueron de 583 mg/L para T1 y $517 \mathrm{mg} / \mathrm{L}$ para T2; valores que no cumplen con el límite permisible para la descarga en cuerpos de aguas naturales, por lo que se hace necesario realizar un postratamiento del efluente del reactor para que este logre cumplir con los valores normativos [12].

En la tabla 3 se pueden observar los valores promedios de nitrógeno total Kjeldahl y nitrógeno amoniacal a la entrada, fin de cada fase y la salida del reactor, para ambos tratamientos. La concentración de NTK fue $100,67 \mathrm{mg} / \mathrm{L}$ a la salida del reactor para el tratamiento $\mathrm{T} 1$ y $62,22 \mathrm{mg} / \mathrm{L}$ para el tratamiento T2; mientras que para el nitrógeno amoniacal los valores fueron de 69,91 y 67,42 $\mathrm{mg} / \mathrm{L}$ para $\mathrm{T} 1$ y $\mathrm{T} 2$, respectivamente.

En la tabla 4 se presentan las concentraciones de nitritos y nitratos durante las diferentes fases del proceso de tratamiento. Las concentraciones de nitritos y nitratos para ambos tratamientos al final de la fase aeróbica, fueron de 28,8 y $65,0 \mathrm{mg} / \mathrm{L}$ para el tratamiento $\mathrm{T} 1$; mientras que para el tratamiento $\mathrm{T} 2$ las concentraciones obtenidas fueron de 48,0 y $65,0 \mathrm{mg} / \mathrm{L}$, respectivamente. Durante la fase anóxica y debido al proceso de desnitrificación, las concentraciones de nitritos y nitratos disminuyeron para T1 a valores de 
Tabla 3. Valores promedios de las concentraciones de NTK y N-NH $4_{4^{+}}$en cada tratamiento aplicado en el reactor.

\begin{tabular}{|c|c|c|c|}
\hline \multirow{4}{*}{ Variable } & Fase & $\begin{array}{c}\text { T1 } \\
\text { Media } \pm \text { DE }\end{array}$ & $\begin{array}{c}\text { T2 } \\
\text { Media } \pm \text { DE }\end{array}$ \\
\hline \multirow{4}{*}{ NTK $(\mathrm{mg} / \mathrm{L})$} & Carga & $434,2 \pm 188,2$ & $649,5 \pm 169,7$ \\
\cline { 2 - 4 } & Fin Anaeróbica & $275,4 \pm 59,1$ & $573,0 \pm 183,8$ \\
\cline { 2 - 4 } & Fin Aeróbica & $201,1 \pm 75,9$ & $123,8 \pm 73,8$ \\
\cline { 2 - 4 } & Fin Anóxica & $147,9 \pm 57,7$ & $88,57 \pm 50,7$ \\
\cline { 2 - 4 } & Salida & $100,7 \pm 55,3$ & $62,22 \pm 17,2$ \\
\hline \multirow{5}{*}{$\mathrm{N}-\mathrm{NH}_{3}(\mathrm{mg} / \mathrm{L})$} & Carga & $214,9 \pm 93,2$ & $408,9 \pm 113,4$ \\
\cline { 2 - 4 } & Fin Anaeróbica & $136,3 \pm 29,3$ & $335,6 \pm 115,2$ \\
\cline { 2 - 4 } & Fin Aeróbica & $99,6 \pm 37,6$ & $106,2 \pm 100,3$ \\
\cline { 2 - 4 } & Fin Anóxica & $102,7 \pm 40,1$ & $71,3 \pm 37,5$ \\
\cline { 2 - 4 } & Salida & $69,9 \pm 38,4$ & $67,4 \pm 38,8$ \\
\hline
\end{tabular}

DE: Desviación estándar

Tabla 4. Valores promedios de las concentraciones de $\mathrm{N}-\mathrm{NO}_{2^{-1}}, \mathrm{~N}-\mathrm{NO}_{3^{-}}$y NT en cada tratamiento aplicado en el reactor.

\begin{tabular}{|c|c|c|c|}
\hline Variable & Fase & $\begin{array}{c}\text { T1 } \\
\text { Media } \pm \text { DE }\end{array}$ & $\begin{array}{c}\text { T2 } \\
\text { Media } \pm \text { DE }\end{array}$ \\
\hline \multirow{5}{*}{$\begin{array}{l}\mathrm{N}-\mathrm{NO}_{2}^{-} \\
(\mathrm{mg} / \mathrm{L})\end{array}$} & Carga & ND & ND \\
\hline & Fin Anaeróbica & ND & ND \\
\hline & Fin Aeróbica & $28,8 \pm 22,5$ & $48,0 \pm 26,8$ \\
\hline & Fin Anóxica & $7,5 \pm 3,5$ & $21,3 \pm 14,4$ \\
\hline & Salida & $7,5 \pm 3,5$ & $22,5 \pm 15,0$ \\
\hline \multirow{5}{*}{$\begin{array}{l}\mathrm{N}-\mathrm{NO}_{3}^{-} \\
\left(\mathrm{mg} / \mathrm{L}^{-}\right.\end{array}$} & Carga & ND & ND \\
\hline & Fin Anaeróbica & ND & ND \\
\hline & Fin Aeróbica & $65,0 \pm 43,6$ & $65,0 \pm 33,5$ \\
\hline & Fin Anóxica & $17,5 \pm 10,6$ & $24,0 \pm 16,4$ \\
\hline & Salida & $17,5 \pm 10,6$ & $13,0 \pm 6,7$ \\
\hline \multirow{5}{*}{$\begin{array}{c}N T \\
(\mathrm{mg} / \mathrm{L})\end{array}$} & Carga & $434,2 \pm 188,2$ & $649,5 \pm 169,7$ \\
\hline & Fin Anaeróbica & $275,4 \pm 59,1$ & $573,0 \pm 183,8$ \\
\hline & Fin Aeróbica & $277,8 \pm 125,2$ & $236,8 \pm 100,7$ \\
\hline & Fin Anóxica & $176,6 \pm 75,6$ & $133,8 \pm 81,4$ \\
\hline & Salida & $130,7 \pm 73,8$ & $97,7 \pm 38,9$ \\
\hline
\end{tabular}

DE: Desviación estándar. ND: No detectable. Límite de detección= $1 \mathrm{mg} / \mathrm{L}$. 
7,5 y $17,5 \mathrm{mg} / \mathrm{L}$, respectivamente; y para $T 2$ a concentraciones de 21,3 y $24,0 \mathrm{mg} / \mathrm{L}$.

En lo referente al nitrógeno total, se obtuvieron valores promedio a la salida del reactor de $130,7 \mathrm{mg} / \mathrm{L}$ para el tratamiento $\mathrm{T} 1, \mathrm{y}$ de 97,7 $\mathrm{mg} / \mathrm{L}$ para el tratamiento $\mathrm{T} 2$, logrando con respecto a los valores de la entrada remociones superiores al 69 y $84 \%$, respectivamente (tabla 5). Se obtuvieron eficiencias de remoción de nitrógeno total Kjeldahl superiores al $75 \%$ para el tratamento $\mathrm{T} 1$, y al $90 \%$ para $\mathrm{T} 2$.

Encuantoalaremoción de nitrógeno amoniacal, los porcentajes obtenidos son mayores a los reportados por Rodríguez y Peñuela [23], quienes aplicaron tratamientos con sistemas SBR a efluentes de matadero de reses usando un tiempo de retención hidráulica de 8 horas, con lo que se lograron remociones de $70,5 \%$, comprobándose que mientras más largo sea el tiempo de reacción durante las fases aeróbica/ anaeróbica (TRH), mayores remociones se alcanzan del nitrógeno amoniacal.

Los resultados obtenidos en esta investigación fueron inferiores a los reportados por Jia et al. [24], quienes, a pesar de aplicar un tiempo de retención hidráulica menor a 12 horas en el tratamiento de efluentes de matadero de reses usando un sistema SBR, lograron una remoción del $92 \%$ de nitrógeno amoniacal. Se infiere que esta diferencia se deba a la aplicación de una prefermentación al efluente cárnico. Merzouki et al. [25], al aplicar una prefermentación, lograron disminuir significativamente el contenido de nitrógeno orgánico presente en el efluente de un matadero desde 690 a 50 $m g \cdot \mathrm{L}^{-1}$, manifestándose en un incremento del $\mathrm{N}-\mathrm{NH}_{4^{+}}$y en mejoras en la tratabilidad biológica del efluente.

Paralasdoscondicionesaplicadas, seobtuvieron eficiencias de nitrificación superiores al 60 y $80 \%$; y eficiencias de desnitrificación superiores al $85 \%$ y al $95 \%$, para los tratamientos T1 y $T 2$, respectivamente, obteniéndose diferencias significativas entre los dos tratamientos ( $p \leq$ $0,05)$. Se infiere que estas diferencias se hayan debido a dos factores principalmente, uno de estos el aumento del tiempo de retención hidráulica, que conllevó a mayores tiempos de reacción durante las fases aeróbica y anóxica; y el segundo factor, la relación C/N manejada en ambos tratamientos.

Dentro de los parámetros que afectan los procesos de nitrificación y desnitrificación,

Tabla 5. Valores promedios de remoción de NTK, NT, y eficiencias de nitrificación y desnitrificación en cada tratamiento aplicado en el reactor.

\begin{tabular}{|c|c|c|}
\hline Variable & $\begin{array}{c}\text { T1 } \\
\text { Media } \pm \text { DE }\end{array}$ & Media \pm DE \\
\hline Remoción de NTK $(\%)$ & $76,8^{\mathrm{b}} \pm 14,6$ & $90,4^{\mathrm{a}} \pm 4,8$ \\
\hline Remoción de NT (\%) & $69,9^{\mathrm{b}} \pm 6,1$ & $85,0^{\mathrm{a}} \pm 7,7$ \\
\hline Eficiencia de Nitrificación (\%) & $63,8^{\mathrm{b}} \pm 20,5$ & $83,6^{\mathrm{a}} \pm 7,3$ \\
\hline Eficiencia de Desnitrificación (\%) & $87,9^{\mathrm{a}} \pm 2,8$ & $96,2^{\mathrm{a}} \pm 1,2$ \\
\hline
\end{tabular}

prueba de Tukey $(p \leq 0,05)$ 
el más crítico es la relación entre la demanda química de oxígeno y el nitrógeno en el efluente (DQO/N). Particularmente para el proceso de la nitrificación, el contenido de carbono/nitrógeno del agua residual determina la competencia en el crecimiento que se establece entre las poblaciones de microorganismos autótrofas y heterótrofas, por lo que a elevadas relaciones $\mathrm{C} / \mathrm{N}$, el proceso de nitrificación se inhibe y la concentración de nitrato cae a cero [26].

Mientras que en lo referente al proceso de desnitrificación, es muy importantela existencia de una relación adecuada de C/N, y una fuente de carbono fácilmente biodegradable para que el proceso de desnitrificación se lleve a cabo con eficacia.

Esto es debido a la actividad microbiológica del sistema, en el que diversos grupos de bacterias compiten con las desnitrificantes para utilizar el nitrato y transformarlo en otros productos que no son nitrógeno gaseoso. A medida que la relación C/N es menor, los microorganismos requieren un mayor tiempo para transformar la materia orgánica que no se degrada fácilmente [27].

Algunos autores, como Roy et al. [28] y Kundu et al. [16] demostraron en sus estudios realizados a tratamientos de efluentes camaroneros y de matadero de reses mediante la aplicación de un SBR, que para lograr remociones máximas de nitrógeno amoniacal, nitritos y nitratos, la relación óptima C/N era de 10:1 a 12:1.

Roy et al. [28] con una relación $\mathrm{C} / \mathrm{N}$ igual a 10:1, reportaron remociones del $100 \%$ de nitrógeno amoniacal $\left(\mathrm{N}-\mathrm{NH}_{3}\right), 91 \%$ de nitritos ( $\mathrm{N}-\mathrm{NO}_{2-}$ ) y $99 \%$ de nitratos ( $\left.\mathrm{N}-\mathrm{NO}_{3-}\right)$, porcentajes superiores a los obtenidos en esta investigación. Por otra parte, Kundu et al. [16] trabajaron con relaciones $\mathrm{C} / \mathrm{N}$ de 10:1 a 12:1, logrando altas remociones de $96,2 \%$ de $\mathrm{N}-\mathrm{NH} 3$ y de $84,4 \%$ de nitrógeno total (NT) con respecto a las obtenidas en el presente estudio, a pesar de que trabajaron con un tiempo de retención hidráulica de 8 horas, menor al aplicado en los tratamientos de esta investigación.

Las relaciones $\mathrm{C} / \mathrm{N}$ obtenidas en la presente investigación para los dos tratamientos aplicados T1 y T2, fueron de 38:1 y 26:1, respectivamente, que en comparación con lo establecido por Roy et al. [28] y Kundu et al. [19], resultaron ser muy elevadas, siendo el menor valor obtenido para el tratamiento T2 el más favorecedor en cuanto a remoción de nitrógeno se refiere, alcanzando porcentajes de remoción de nitrógeno amoniacal y nitritos-nitratos iguales a 83,6 y $96,1 \%$, respectivamente.

Por ser el pH y la alcalinidad en los sistemas biológicos parámetros vitales para la realización de los procesos de reducción biológica de nutrientes y materia orgánica, se muestra en las figuras 3 y 4 el comportamiento de las concentraciones de las diferentes especies de nitrógeno, $\mathrm{NTK}, \mathrm{N}-\mathrm{NH}_{4^{+},} \mathrm{N}-\mathrm{NO}_{3^{-},} \mathrm{N}-\mathrm{NO}_{2^{-}}$ y la evolución de las variables de control operacional $\mathrm{pH}$ y alcalinidad total, durante un ciclo operacional de los tratamientos T1 y T2, respectivamente.

En la figura 3, que hace referencia al primer tratamiento aplicado, se puede observar una reducción del $\mathrm{pH}$ en el transcurso de la fase anaeróbica. Durante esta fase, los microorganismos se encargan de oxidar tanto la materia orgánica presente en el medio, así como una parte del nitrógeno que es removido por asimilación, y la otra es convertida a través de la descomposición bacteriana e hidrólisis, en nitrógeno amoniacal [27]. Este fenómeno de reducción de $\mathrm{pH}$ en la fase anaeróbica lo describen algunos autores, como Kundu 
et al. [16], quienes establecen que durante la oxidación de la materia orgánica ocurren disminuciones en este parámetro.

Se puede observar que durante la fase de aireación ocurren simultáneamente tanto un aumento notable del $\mathrm{pH}$, como un consumo de alcalinidad, durante una fase en la que el $\mathrm{pH}$ teóricamente debería decrecer como consecuencia del proceso de nitrificación que genera iones hidrógeno. Sin embargo, la suficiente alcalinidad contenida en el medio, permitió contrarrestar dichos cambios en el $\mathrm{pH}$ y que lo mantuviera dentro del rango establecido como óptimo [27] $(7,2 \leq \mathrm{pH} \leq 9,0)$, permitiendo que el proceso de nitrificación no se inhibiera y ocurriera en mayor grado. Resultados similares fueron reportados por Carrasquero et al. [29] tratando efluentes de una tenería en un SBR.

Durante las dos primeras fases, empezando por la etapa anaeróbica, el 36,6 \% del nitrógeno total Kjeldahl (NTK) y nitrógeno amoniacal (N$\mathrm{NH}_{3}$ ) fueron removidos del sistema. Se infiere que esta remoción ocurrió como consecuencia de los procesos de asimilación bacteriana y catabolismo, mediante los cuales una fracción removida de nitrógeno orgánico fue convertida en biomasa heterótrofa y autótrofa.

En la fase aeróbica se observó que ocurrió una disminución del 26,3\% tanto de nitrógeno total Kjeldahl (NTK) como de nitrógeno amoniacal $\left(\mathrm{N}-\mathrm{NH}_{4^{+}}\right)$, producto de la transformación del nitrógeno orgánico en nitrógeno amoniacal ( $\mathrm{N}$ $\mathrm{NH}_{4^{+}}$) mediante los procesos de amonificación y nitrificación, en el que el nitrógeno amoniacal se transformó en nitritos $\left(\mathrm{N}^{-\mathrm{NO}_{3}}\right.$ ) y nitratos $\left(\mathrm{N}-\mathrm{NO}_{2}-\right.$ ), observándose un aumento en la concentración de estas especies en el final de la fase. Sin embargo, las concentraciones tanto de NTK como de $\mathrm{N}-\mathrm{NH}_{4^{+}}$no fueron removidas en su totalidad, resultando para el tratamiento T1 una eficiencia de nitrificación de $63,8 \%$. Se infiere que la ocurrencia de una nitrificación incompleta pudo ser causada por el alto contenido de nitrógeno amoniacal en el efluente industrial y un reducido período de reacción durante la fase aeróbica, así como por una alta relación $\mathrm{C} / \mathrm{N}$ del efluente.

Durante la fase anóxica se observó que como resultado del proceso de desnitrificación,
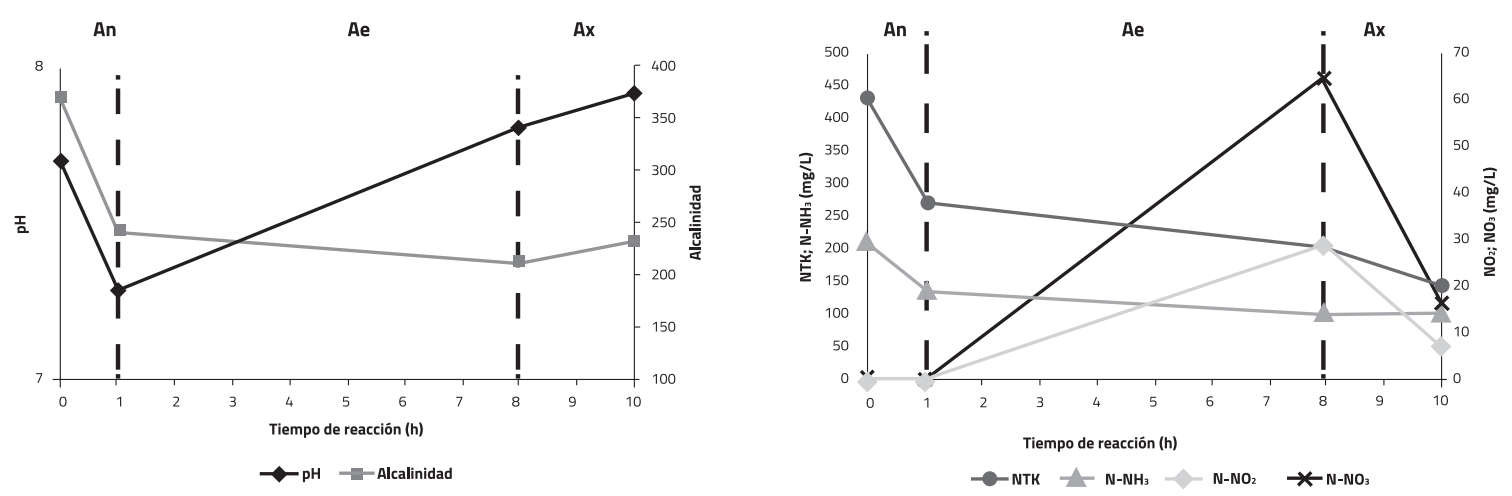

Figura 3. Perfil de las concentraciones de $\mathrm{NTK}, \mathrm{N}-\mathrm{NH}_{3^{\prime}} \mathrm{N}-\mathrm{NO}_{2^{-}}, \mathrm{N}-\mathrm{NO}_{3^{-}}, \mathrm{pH}$ y alcalinidad total durante el ciclo de operación del reactor para T1 
las concentraciones de nitritos y nitratos se redujeron desde 28,75 y $65,00 \mathrm{mg} / \mathrm{L}$, respectivamente, hasta $7,5 \mathrm{mg} / \mathrm{L}$ y $17,50 \mathrm{mg} / \mathrm{L}$ para una eficiencia de desnitrificación del 87,9\%. El nitrógeno total Kjeldahl (NTK) presentó una ligera disminución de su concentración durante esta fase, y el contenido de nitrógeno amoniacal ( $\mathrm{N}-\mathrm{NH}_{4^{+}}$) permaneció casi constante; esta fracción de $\mathrm{N}-\mathrm{NH}_{\mathrm{L}^{+}}$que no se transformó en nitritos y nitratos, abandonó el sistema de tratamiento sin haber sido eliminado, con lo que se logró, por consiguiente, una remoción del $69,90 \%$ de nitrógeno total.

En cuanto al comportamiento de las variables operacionales durante esta última fase, se observó un leve aumento del $\mathrm{pH}$ $y$, por consiguiente, una recuperación de la alcalinidad en el sistema, tal y como lo afirma Carrasquero et al. [28], quienes concluyeron que la desnitrificación libera alcalinidad, por lo que consigue recuperar una parte de la alcalinidad consumida por la nitrificación.

En la figura 4 se muestra el comportamiento de cada una de las variables en estudio, pero para el segundo tratamiento, en el que se presentaron notorias diferencias en la tendencia de las variables de control con respecto al tratamiento $\mathrm{T} 1$.

El comportamiento del $\mathrm{pH}$ fue diferente con respecto a lo obtenido en el tratamiento T1. Durante la etapa anaeróbica se observó que los valores de $\mathrm{pH}$ presentaron ligeros incrementos hasta finalizar la fase debido a la alcalinidad presente en el medio. Por otra parte, las concentraciones de nitrógeno total Kjeldahl (NTK) y nitrógeno amoniacal (N$\mathrm{NH}_{4^{+}}$) fueron reducidas, en un 11,7 y $17,9 \%$, respectivamente, debido a los procesos de asimilación bacteriana y catabolismo, tal como sucedió anteriormente en el tratamiento T1.

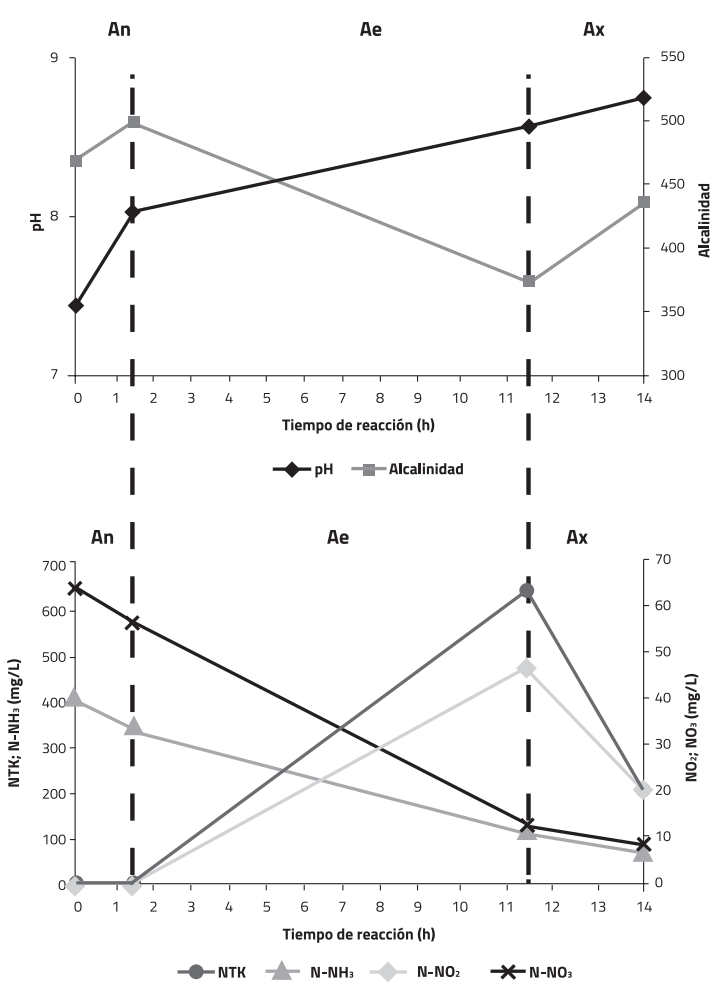

Figura 4. Perfil de las concentraciones de $\mathrm{NTK}, \mathrm{N}-\mathrm{NH}_{3}$ $\mathrm{N}-\mathrm{NO}_{2^{-},} \mathrm{N}-\mathrm{NO}_{3^{-}}, \mathrm{pH}$ y alcalinidad total durante el ciclo de operación del reactor para el tratamiento T2.

Durante la segunda fase del tratamiento, la etapa aeróbica, se obtuvieron disminuciones de NTK y de $\mathrm{N}^{-\mathrm{NH}_{4^{+}}}$de 78,4 y 68,4\%, respectivamente, como producto del proceso de nitrificación, resultando ser remociones superiores a las obtenidas durante la primera condición aplicada; se comprobó que el aumento en el tiempo de reacción durante la aireación logró mayores remociones de $\mathrm{N}-\mathrm{NH}_{4^{+}}$en el sistema, obteniendo por consiguiente la presencia de $\mathrm{N}-\mathrm{NO}_{3^{-}}$y N-NO en concentraciones que se incrementaron hasta el final de la fase.

Durante la fase anóxica se produjo un ligero aumento del $\mathrm{pH}$, una recuperación de la 
alcalinidad consumida durante la fase anterior, y una disminución en la concentración de los nitratos como resultado del proceso de desnitrificación, lo que permitió obtener a la salida del tratamiento concentraciones de nitritos y nitratos iguales a 21,25 y $24,00 \mathrm{mg} / \mathrm{L}$, respectivamente, y se logró una eficiencia de desnitrificación durante esta fase del 96,2 \%, y una remoción de nitrógeno total del 85,0\%.

Para ambos tratamientos se observó, además, que la cantidad de alcalinidad consumida en el proceso de nitrificación fue mayor que la alcalinidad generada en la desnitrificación. Carrasquero et al. [30] y Li e Irvin [31] aseguraron que la variación de la alcalinidad a lo largo del proceso de tratamiento podría ser utilizado como un indicador del grado de nitrificación/desnitrificación obtenido en el sistema.

En relación con el fósforo total, los resultados obtenidos en porcentajes de remoción durante los dos tratamientos se presentan en la tabla 6 , con el respectivo valor de concentración a la entrada del reactor y lo obtenido a la salida del mismo.
El tratamiento T1 mostró un porcentaje de remoción del $29,09 \%$, con una entrada de 10,01 $\mathrm{mg} / \mathrm{L}$ de fósforo total y una concentración de $7,19 \mathrm{mg} / \mathrm{L}$ a la salida. Mientras que durante el tratamiento $\mathrm{T} 2$, el porcentaje de remoción fue mayor, reportándose un $44,07 \%$ de fósforo removido, con una entrada de $11,62 \mathrm{mg} / \mathrm{L}$ y una concentración a la salida de $6,40 \mathrm{mg} / \mathrm{L}$. Ambos tratamientos presentaron diferencias estadísticas $(p \leq 0,05)$ que son atribuidas a la variación en el tiempo de retención hidráulica.

Para ambos tratamientos, el reactor por carga secuencial logró disminuir las concentraciones hasta cumplir con el requisito de contenido de fósforo total menor de $10 \mathrm{mg} / \mathrm{L}$, según lo establecido por la norma venezolana de descarga a los cuerpos de agua [12].

\section{CONCLUSIONES}

Los dos tratamientos aplicados al efluente industrial de matadero de reses en el reactor por carga secuencial (SBR) permitieron la remoción simultánea de nutrientes y materia orgánica, debido a la flexibilidad del reactor para

Tabla 6. Valores promedios de las concentraciones y porcentajes de remoción de fósforo total (PT) en cada tratamiento aplicado en el reactor.

\begin{tabular}{|c|c|c|c|}
\hline $\begin{array}{c}\text { Variable } \\
\text { (mg/L) }\end{array}$ & Fase & $\begin{array}{c}\text { T1 } \\
\text { Media } \pm \mathbf{D E}\end{array}$ & $\begin{array}{c}\text { T2 } \\
\text { Media } \pm \text { DE }\end{array}$ \\
\hline \multirow{4}{*}{ Fósforo } & Carga & $10,1 \pm 0,3$ & $11,6 \pm 0,7$ \\
\cline { 2 - 4 } & Fin Anaeróbica & $10,8 \pm 0,5$ & $13,5 \pm 0,1$ \\
\cline { 2 - 4 } & Fin Aeróbica & $7,0 \pm 1,7$ & $7,6 \pm 1,7$ \\
\cline { 2 - 4 } & Fin Anóxica & $7,2 \pm 2,1$ & $6,4 \pm 2,9$ \\
\cline { 2 - 4 } & Salida & $7,2 \pm 2,1$ & $6,4 \pm 2,9$ \\
\hline & $29,1^{\text {b }} \pm 19,0$ & $44,1^{\text {a }} \pm 27,7$ \\
\hline
\end{tabular}

Nota: Media seguida por letras distintas en cada fila indica diferencias significativas según la prueba de Tukey $(\mathrm{p} \leq 0,05)$. 
adaptarse a distintas condiciones de operación, obteniendo para los dos tratamientos aplicados, remociones superiores al $95 \%$ para la $\mathrm{DQO}_{T}, 69 \%$ para el NT y $29 \%$ para el PT.

No hubo diferencias significativas ( $p>0,05$ ) para la remoción de $\mathrm{DQO}_{\mathrm{T}}$ durante los dos tratamientos evaluados, por lo cual el TRH no afectó la remoción de estos parámetros. Caso contrario ocurrió con la remoción de NTK, NT y PT, donde sí resultaron diferencias significativas entre los tratamientos aplicados. Existieron diferencias significativas $(p \leq$ 0,05 ) en las eficiencias de nitrificación y desnitrificación para los dos tratamientos evaluados, por lo que la variación en el TRH de 12 a $15 \mathrm{~h}$ afectó estos procesos. El aumento en el tiempo de retención hidráulica conllevó a un aumento tanto en la eficiencia de nitrificación como en la eficiencia de desnitrificación, por el alargamiento de las fases aeróbica y anóxica en la etapa de reacción.

El tratamiento T2, con un tiempo de retención hidráulica (TRH) de 15 horas y una secuencia de operación anaeróbica-aeróbica y anóxica, generó mayores rendimientos en cuanto a la eliminación simultánea de DQO $(95,39 \%)$, NTK $(90,42 \%)$, NT $(84,95 \%)$ y PT $(44,07 \%)$ que el tratamiento $\mathrm{T} 1$, que tuvo un $\mathrm{TRH}$ igual a 11 horas.

\section{BIBLIOGRAFÍA}

[1] Corbitt, R. (2003). Manual de referencia de la Ingeniería Medioambiental. España: Editorial McGraw-Hill. 1.608 p.

[2] Flapper, T., Ashbolt, N., Lee, A. y Neil, 0. (2001). From the laboratory to full-scale SBR operation: treating high strength and variable industrial wastewaters. En: Water Science and Technology, Vol. 43(3), pp. 347-365.

[3] Pabón, S. y Suárez, J. (2009). Arranque y operación a escala real de un sistema de tratamiento de lodos activos para aguas residuales de matadero. En: Revista Ingeniería e Investigación, Vol. 29(2), pp. 53-58.

[4] Padilla, E., López, A. y Gallardo, J. (2011). Evaluation of stability factors in the anaerobic treatment of slaughterhouse water. En: Journal Bioremediation and Biodegradation, Vol. 2(1), pp. 1-5.

[5] López, A., De la Barrera, J., Vallejo, R. y Barahona, C. (2008). Estudio comparativo entre un proceso fisicoquímico y uno biológico para tratar agua residual de rastro. En: Interciencia. Vol. 33(7), pp. 490-495.

[6] Massé, D. y Masse, L. (2000). Treatment of slaughterhouse wastewater in anaerobic sequencing batch reactors. En: Agriculture and Agri-Food Canada contribution. Vol. 42(3), pp.131-137.

[7] Filali, M., Auriol, M., Tyagi, R. y Surampalli, R. (2004). Treatment of slaughterhouse wastewater in a sequencing batch reactor: simulation vs. experimental studies. En: Environmental Technology. Vol. 25(1), pp. 23-28.

[8] Li, J., Healy, M., Zhan, X., Norton, D. y Rodgers, M. (2008a). Effect of aeration rate on nutrient removal from slaughterhouse wastewater in intermittently aerated sequencing batch 
reactors. En: Water Air Soil Pollut. Vol. 192, pp. 251-261.

[9] Li, J., Healy, M., Zhan, X. y Rodgers, M. (2008b). Nutrient removal from slaughterhouse wastewater in an intermittently aerated sequencing batch reactor. En: Bioresoure Technology, Vol. 99, pp. 7644-7650.

[10] Zhan, X., Healy, M., y Li, J. (2009). Nitrogen removal from slaughterhouse wastewater in a sequencing batch reactor under controlled low DO concentrations. En: Bioprocess Biosystem Engineering, Vol. 32, pp. 607-614.

[11] Farabegoli, G., Caruccí, A., Majone, M. y Rolle, E. (2004). Biological Treatment of tannery wastewater in the presence of chromium. En: Journal of Environmental Management, Vol. 71, pp. 345-349.

[12] Gaceta Oficial de la República de Venezuela. (1995). Normas para la clasificación y el control de la calidad de los cuerpos de agua y de los vertidos líquidos. No. 5021. 18-12-1995. Decreto 883. Venezuela, p. 17.

[13] Uygur, A. (2006). Specific nutrient removal rates in saline wastewater treatment using sequencing batch reactor. En: Process Biochemistry, Vol. 41, pp. 61-66.

[14] Díaz, A., Peña, M., Carrasquero, S., López, F. y Escalera, M. (2012). Remoción de nitrógeno en efluentes de una industria camaronera utilizando un reactor por carga secuencial. En: Ciencia, Vol. 20(2), pp. 77-89.

[15] American Public Health Association (APHA-AWWA-WEF). (2005). Standard
Methods for the Examination of Water and Wastewater. (21th edition). American Public Health Association, Washintong, D.C., U.S.A.

[16] Kundu, P., Debsarkar, A. y Mukherjee, S. (2013). Treatment of slaughterhouse wastewater in a sequencing batch reactor: Performance evaluation and biodegradation kinetics. En: BioMed Research International, Vol. 1, pp. $1-11$.

[17] Ramón, A. y Maldonado J. (2006). Sistema de tratamiento para aguas residuales industriales en mataderos. En: Revista Ambiental Agua Aire y Suelo, Vol. 1(1), pp. 34-47.

[18] Bazrafshan, E., Kord, F., Farzadkia, M., Ownagh, K. y Mahvi, A. (2012). Slaughterhouse wastewater treatment by combined chemical coagulation and electrocoagulation process. En: Plosone, Vol. 7(6), pp. 1-8.

[19] Sunder, G. y Satyanarayan, S. (2013). Efficient treatment of slaughterhouse wastewater by anaerobic hybrid reactor packed with special floating media. En: International Journal of Chemical and Physical Sciences, Vol. 2, pp. 73-81.

[20] Nakhla, G., Al-Sabawi, M., Bassi, A. y Liu, V. (2003). Anaerobic treatability of high oil and grease rendering wastewater. En: Journal of Hazardous Materials, Vol. 102(2-3), pp. 243-255.

[21] Inescop. Centro de Innovación y Tecnología. (2008). Aplicación de bioensayos respirométricos en aguas de tenerías. Departamento del 
Medioambiente de Inescop, Alicante, España, 16 p.

[22] Nacheva, P., Pantoja, M. y Serrano, E. (2011). Treatment of slaughterhouse wastewater in upflow anaerobic sludge blanket reactor. En: Waster Science and Technology, Vol. 63(5), pp. 877-884.

[23] Rodríguez, D. y Peñuela, G. (2011). Molecular distribution of wastewater from a meat by-products processing company, treated using a batch system (SBR). En: Revista de Química Teórica y Aplicada, Vol. 68, pp. 459-466.

[24] Jia, Y., Gao, C., Zhang, L. y Jiang, G. (2012). Effect of pre-fermentation and influent temperature on the removal efficiency of $\mathrm{COD}, \mathrm{NH}_{4^{+}}-\mathrm{N}$ and PO43-P in slaughterhouse wastewater by using SBR. En: Energy Procedia, Vol. 16, pp.1964-1971.

[25] Merzouki, M., Bernet, N., Delgenès, J., Benlemlih, M. (2005). Effect of prefermentation on denitrifying phosphorus removal in slaughterhouse wastewater. En: Bioresource Technology, 96:1317-1322.

[26] Cheng, S., y Chen, W. (1994). Organic carbon supplements influencing performance of biological nitrification in a fluidized bed reactor. En: Water Science Techonoloy, Vol. 30, pp. 131-142.

[27] Barajas, M. (2002). Eliminación biológica de nutrientes en un reactor biológico secuencial. Trabajo doctoral de Ciencias Biológicas. Departamento de Ingeniería Hidráulica, Marina y Ambiental.
Universidad Politécnica de Cataluña, Barcelona, España. 345 p.

[28] Roy, D., Hassan, K., Boopathy, R. (2010). Effect of carbon to nitrogen (C:N) ratio on nitrogen removal from shrimp production wastewater using sequencing batch reactor. En: J Ind Microbiol Biotechnol, Vol. 37, pp. 1105-1110.

[29] Carrasquero, S., Pire, M., Rincón, A., Díaz, A. (2014). Monitoreo de la remoción biológica de nitrógeno en efluentes de tenerías usando un reactor por carga secuencial. En: Ingeniería, Investigación y Tecnología. Vol. 15(2), pp. 287-298.

[30] Carrasquero, S., Pire, M., Colina, G., Mas y Rubí, M., Martínez, D., Díaz, A. (2013). Tasas de nitrificación y desnitrificación durante el tratamiento biológico de efluentes de tenerías en un reactor por carga secuencial. En: Boletín del Centro de Investigaciones Biológicas, Vol. 47(3), pp. 220-234.

[31] Li, B., Irvin, S. (2007). The comparison of alkalinity and ORP as indicators for nitrification and denitrification in a sequencing batch reactor (SBR). En: Biochemical Engineering Journal, Vol. 34, pp. 248-255. 